\title{
SARS-CoV-2 and Probable Lung Cancer Risk
}

\author{
sajad khiali $^{1}$, Afra Rezagholizadeh ${ }^{1}$, and Taher Entezari-Maleki ${ }^{1}$ \\ ${ }^{1}$ Affiliation not available
}

July 21, 2020

\begin{abstract}
As of 16 July 2020, globally about 13.4 million cases of coronavirus disease 2019 (COVID-19) have been confirmed with nearly 600000 deaths. One of the main concerns about COVID-19 is long-term complications. Viruses account for about one-fifth of human cancer cases $[1,2]$. Herein, three possible mechanisms of the novel severe acute respiratory syndrome coronavirus 2 (SARS-CoV-2) as an oncogenic virus in the lung have been discussed briefly.
\end{abstract}

\section{SARS-CoV-2 and Probable Lung Cancer Risk}

Sajad Khiali Pharm.D. ${ }^{1}$, Afra Rezagholizadeh Pharm.D. ${ }^{1}$, Taher Entezari-Maleki Pharm.D, $\mathrm{PhD}^{1,} \mathbf{2}^{*}$

1-Department of clinical pharmacy, Faculty of pharmacy, Tabriz University of Medical Sciences, Tabriz, Iran.

2-Cardiovascular Research Center, Tabriz University of Medical Sciences, Tabriz, Iran.

\section{Corresponding author:}

Taher Entezari-Maleki Pharm.D., PhD

Associated Professor of Clinical Pharmacy

tentezari@gmail.com or entezarim@tbzmed.ac.ir

Department of clinical pharmacy, Faculty of pharmacy, Tabriz University of Medical Sciences, Tabriz, Iran.

As of 16 July 2020, globally about 13.4 million cases of coronavirus disease 2019 (COVID-19) have been confirmed with nearly 600000 deaths. One of the main concerns about COVID-19 is long-term complications. Viruses account for about one-fifth of human cancer cases [1,2]. Herein, three possible mechanisms of the novel severe acute respiratory syndrome coronavirus 2 (SARS-CoV-2) as an oncogenic virus in the lung have been discussed briefly.

First, available data have been shown a chronic involvement of pulmonary tissue in patients with the previous zoonotic coronaviruses mainly severe acute respiratory syndrome coronavirus (SARS-CoV) and Middle East respiratory syndrome coronavirus (MERS-CoV). ${ }^{2-4}$ For example, a 15-year follow-up of 71 patients with SARS-CoV infection showed a chronic involvement of lung tissues with interstitial abnormalities [3]. Das et al. in a study of 36 recovered patients with MERS-CoV infection with a median follow-up of 43 days (ranged from 32-320 days) showed radiographic findings indicating pulmonary fibrosis in about one-third of patients [4]. Recently, Spagnolo et al. are presumed pulmonary fibrosis as a long-term complication of COVID-19 [2]. Mounting evidence showed a direct link as well as common cellular processes and molecular pathways between pulmonary fibrosis and lung cancer. Pulmonary fibrosis is a risk factor for developing lung cancer, and the prevalence of lung cancer in patients with idiopathic pulmonary fibrosis ranges from $2.7 \%$ to $48 \%$ [5]. For the second mechanism, COVID-19 hyperinflation and cytokines storm induced pathologic changes should 
be considered as well. Also, the pro-inflammatory cytokines such as interleukin 1 (IL-1), IL-6, and tumor necrosis factor- $\alpha$ (TNF- $\alpha$ ), which play a key role in COVID-19 related hyper-inflammation pathogenesis, are involved in the tumor promotion and cell transformation of lung cancer pathogenesis. Hyperinflammation is a critical response by the host from the first stages of cancer development. Besides, chronic inflammation not only has a critical function in tumorigenesis, but also could make an ideal environment for carcinogenesis $[6,7]$. Third, similar to patients suffering from non-small-cell lung carcinoma (NSCLC), a down-regulation of angiotensin-converting enzyme 2 (ACE2) occurs after COVID-19 ensuing. ACE2 gene has been confirmed as the functional receptor for SARS-CoV-2, and it has a crucial role in virus entry into the target cell. It degrades angiotensin II (Ang II) and generates angiotensin (1-7). Considerable evidence indicated that the renin-angiotensin system (RAS) components including ACE2, Ang (1-7), and Ang II play a key role in malignancies from the early stages. Low levels of ACE2 expression has been demonstrated as an indicator of malignancies and poor prognostic cancers. ACE2 has an inhibitory effect on cancer cell proliferation, metastasis, invasion, migration, and angiogenesis, especially in NSCLC. Additionally, Ang (1-7) reduces lung cancer cell proliferation and angiogenesis as well and also has an inhibitory effect on A549 human lung adenocarcinoma cells. On the contrary, angiotensin II/ angiotensin II type I receptor (AT1R) axis has a regulatory effect on almost every feature of cancer, including angiogenesis, cell proliferation, dissemination, desmoplasia, tumor growth, cancer-related inflammation induction and promoting immunosuppressive milieu, and tumor progression[8-10].

We hypothesized that SARS-CoV-2 infection could be a trigger for lung cancer. Considering the large number of infected individuals and lack of data regarding postinfection immunity, even a weak association of SARS-CoV-2 infection with lung cancer should be considered in public health policy and evaluated in the future studies.

Funding: None

Conflicting interest: None

Disclosure: The authors have nothing to disclose.

\section{References:}

1. World Health Organization. Coronavirus disease 2019(COVID-19) Situation Report. https://www.who.int/docs/default-source/coronaviruse/situation-reports/20200716-covid-19-sitrep178.pdf?sfvrsn=28ee165b_2

2. Spagnolo P, Balestro E, Aliberti S, et al. Pulmonary fibrosis secondary to COVID-19: a call to arms? [published online ahead of print, 2020 May 15]. Lancet Respir Med . 2020;S2213-2600(20)30222-8.

3. Zhang P, Li J, Liu H. Long-term bone and lung consequences associated with hospital-acquired severe acute respiratory syndrome: a 15-year follow-up from a prospective cohort study. Bone Res . 2020;8:8.

4. Das KM, Lee EY, Singh R. Follow-up chest radiographic findings in patients with MERS-CoV after recovery. Indian J Radiol Imaging . 2017;27:342-349.

5. Ballester B, Milara J, Cortijo J. Idiopathic Pulmonary Fibrosis and Lung Cancer: Mechanisms and Molecular Targets. Int J Mol Sci . 2019 Feb; 20(3): 593.

6. Lance L. Munn. Cancer and inflammation. Wiley Interdiscip Rev Syst Biol Med . 2017 Mar; 9(2): 10.1002/wsbm.1370.

7. O'Callaghan DS, O'Donnell D, O'Connell F, O'Byrne KJ. The role of inflammation in the pathogenesis of non-small cell lung cancer. J THORAC ONCOL . 2010;5(12):2024-36.

8. Xu J, Fan J, Wu F, et al. The ACE2/Angiotensin-(1-7)/Mas Receptor Axis: Pleiotropic Roles in Cancer. FRONT PHYSIOL . 2017;8:276. 
9. Sommerstein R, Kochen MM, Messerli FH, Gräni C. Coronavirus Disease 2019 (COVID 19): Do Angiotensin-Converting Enzyme Inhibitors/Angiotensin Receptor Blockers Have a Biphasic Effect? J. Am. Heart Assoc . 2020;9(7):e016509.

10. Pinter M, Jain RK. Targeting the renin-angiotensin system to improve cancer treatment: Implications for immunotherapy. Sci. Transl. Med . 2017;9(410):eaan5616. 